\title{
INTERVAL TYPE-2 FUZZY LOGIC FOR FIRE OUTBREAK DETECTION
}

\author{
Uduak Umoh ${ }^{1}$, Udoinyang G. Inyang ${ }^{2}$, and Emmanuel E. Nyoho ${ }^{3}$ \\ 1,2,3 Department of Computer Science, University of Uyo, PMB 1017, Akwa Ibom State, \\ 52003, Nigeria
}

\begin{abstract}
This paper employed an Interval Type-2 Fuzzy Logic (IT2FL) in the detection of fire outbreak using environmental parameters. Fire outbreak data capturing device (FODCD) is developed and used in capturing environmental parameter values. The FODCD is comprised of DHTIl temperature sensor, $M Q-2$ smoke sensor, LM393 Flame sensor, and ESP8266 Wi-Fi module connected to Arduino nano v3.0.board. An android application is developed using Android Studio 3.0 to test the functionality of the system. Experimental result indicates that, with the temperature value of $40.657^{\circ} \mathrm{C}$, Smoke value of $77.86 \%$, and Flame value of $762.95 \mathrm{ppm}$ (part per million), with a threshold $T=0.8$, the IT2FL predicted fire outbreak at $0.778(78 \%)$. IT2FL is evaluated using the True Positive Rate (TPR), False Positive Rate (FPR), Accuracy, Error Rate (ER), Precision, and Recall performance metrics. The performance result shows that, the system performs optimally when threshold $T$ is kept between an optimal range of 0.8 and 0.85 .
\end{abstract}

\section{KEYWORDS}

Interval Type-2 Fuzzy Logic, Fire Outbreak Detection, Environmental Parameter, Type Reduction Algorithm

\section{INTRODUCTION}

In recent years, there is great interest in Fuzzy Control (Ross, 2004). The growing popularity of fuzzy control can be attributed to the ability of Fuzzy Logic controllers to provide a vehicle whereby engineers can incorporate human reasoning in the control algorithm. Fuzzy control is a control method based on fuzzy logic (Jantzen, 1999), (Bezdek, 1993). The design of a fuzzy logic system is not based on mathematical modeling process rather it is a non-linear mapping based on expert system process called fuzzy inference. Type-2 Fuzzy logic has the capacity to minimize the effect of uncertainties unlike type-1 fuzzy logic systems (Hagras, 2007), (Wu and Tan, 2006). Fuzzy logic was introduced by Zadeh in 1965 (Zadeh, 1965). Fuzzy Logic is an expert system algorithm that extends the conventional two-valued logic by adding the intermediate values between absolute true and absolute false. A Fuzzy Logic System (FLS) is a non-linear mapping of an input (fuzzy variables) data into an output data (called crisp output). The objective of fuzzy logic is to map crisp input into crisp output (Chu, 2010). Fuzzy logic-based controller's design implements human reasoning that is programmed into membership functions, fuzzy rules, and fuzzy inference.

Fuzzy controllers are able to replace human experts by making effective decisions on the basis of linguistic information such as linguistic variable, and linguistic terms. One of the applications of fuzzy controller is in the area of fire outbreak detection. Fire can be defined as a combustion process resulting in light, smoke, heat, flame and various hazardous gases (Sharma, 2016). The three major 
components of fire are Heat, oxygen and fuel. The proportion of these three components determines the nature of the fire (Sowah, 2014). Although fire contributes to the development of human society and used for a lot of applications such as cooking, manufacturing and other processes, when fire is out of control (called fire hazard or fire outbreak), this can cause serious effect to human life as well as damage to properties. Fire outbreak can also play a role in damaging our ecological environment.

Fire outbreak detection is the process of sensing of one or more phenomena resulting from fire such as smoke, heat, infrared light radiation or gas. Fire outbreak detection is the act of monitoring environmental properties that could lead to fire outbreak and alerting user of potential fire disaster. In the manual process of fire outbreak detection, fire outbreak is detected by visual observation which is a hazardous job and can put the life of a human being in danger. Thus, in order to detect and prevent fire hazard, an intelligent controller for fire outbreak detection based on interval type- 2 fuzzy logic algorithm is developed. By outsourcing the task of fire outbreak detection in fire-prone areas of a room to this system, fire accident, loss of properties, and loss of lives could be avoided. Traditional fire outbreak detection system uses smoke detectors which is less reliable and results in false alarms. In the traditional system, these fire detectors are unable to respond quickly and reliably in hazardous conditions (Mobin et al, 2016). In (Saeed et al., 2018), a wireless sensor network is proposed using multiple sensors for detecting early house fire.

Unlike the traditional fire outbreak detection devices, this work is based on the design and development of a fire outbreak data capture device for automatic fire outbreak detection by interfacing an interval type-2 fuzzy logic approach in order to reduce false alarms and increase the reliability of the entire system. The system uses an Arduino nano microcontroller unit with smoke sensor, temperature sensor and flame sensor as the detection devices. The system collects the data from sensors and passes into an Interval Type-2 Fuzzy Inference system. Compared with existing fire detection systems, the system can reduce the false alarm rate and reduces the chances of missing fire outbreak detection. The fire outbreak detector can provide continuous monitoring of flammable. Based on these decisions, the system can identify the occurrence of fire outbreak conditions. To avoid unwanted and missing fire alarms, a Mamdani type fuzzy inference mechanism is employed (Hassan et al, 2013). This mechanism provides higher accuracy. In (Singh et al., 2017), an investigation on fire alarm is carried out. The study employed telecommunication technology in real-time detection, capturing and monitoring fire. The system explores two controllers to sends signals to GSM and to turn-on the screen. Arduino board is used and programmed to connect and trigger automatically. In Sarwar et al., (2018), fuzzy logic approach is employed to design an intelligent fire monitoring and warning system (FMWS). The study is built based on tiny, low cost, and very small in size sensors technology. The system is able to identify the true existence of dangerous fire, thus, send alert to fire management system (FMS). Sowah et al use fuzzy logic approach in the design and implementation of a fire detection and control system for automobiles.

Fuzzification is the conversion of raw input values (crisp input) into fuzzy set using linguistic variables and fuzzy membership function (Mendel, 1995). Linguistic variables can be categorized into input variables or output variables. The values of these variables are in the form of words or sentences, rather than numeric values (Mendel, 1995). After this process, an inference will be made based on decision rules. This produces an interval type-2 fuzzy set, which are reduced to a type-1 fuzzy set by a type reduction method called the Karnik Mendel algorithm. Now the fuzzy output is obtained using the process called defuzzification. The output of defuzzification (called the crisp output) will be used with an activation function to make decision about potential fire outbreak. The interval type-2 fuzzy controller will be implemented using Android Studio, XML and Java 
programming language for android devices. The system will be tested experimentally on real world data from fire outbreak data capture device (FODCD) connected to a microcontroller unit.

\section{Proposed Framework for fire Outbreak Detection Based On Interval TYPE-2 FUZZY LOGIC}

A Framework for Fire Outbreak Detection Based on Interval Type-2 Fuzzy Logic Algorithm monitors fire outbreak situation via the use of temperature sensor, smoke sensor and flame sensor attached to an Arduino nano microcontroller unit. The system extracts the data from these sensors and transmits them via an ESP8266 Wifi module to an Interval Type-2 Fuzzy Logic controller module running on an android device. The extracted data (input parameters) are then fuzzified (i.e. converted to an interval type-2 fuzzy set). This fuzzy set is passed to an inference engine which evaluates the fuzzy set against the rules in the rule base and produces another type- 2 fuzzy set. The fuzzy set produced during inference is reduced to type-1 fuzzy set via Karnik-Mendel type reduction algorithm. The reduced set is then defuzzified to give a crisp output. The system framework that depicts the above description is presented in Figure 1.

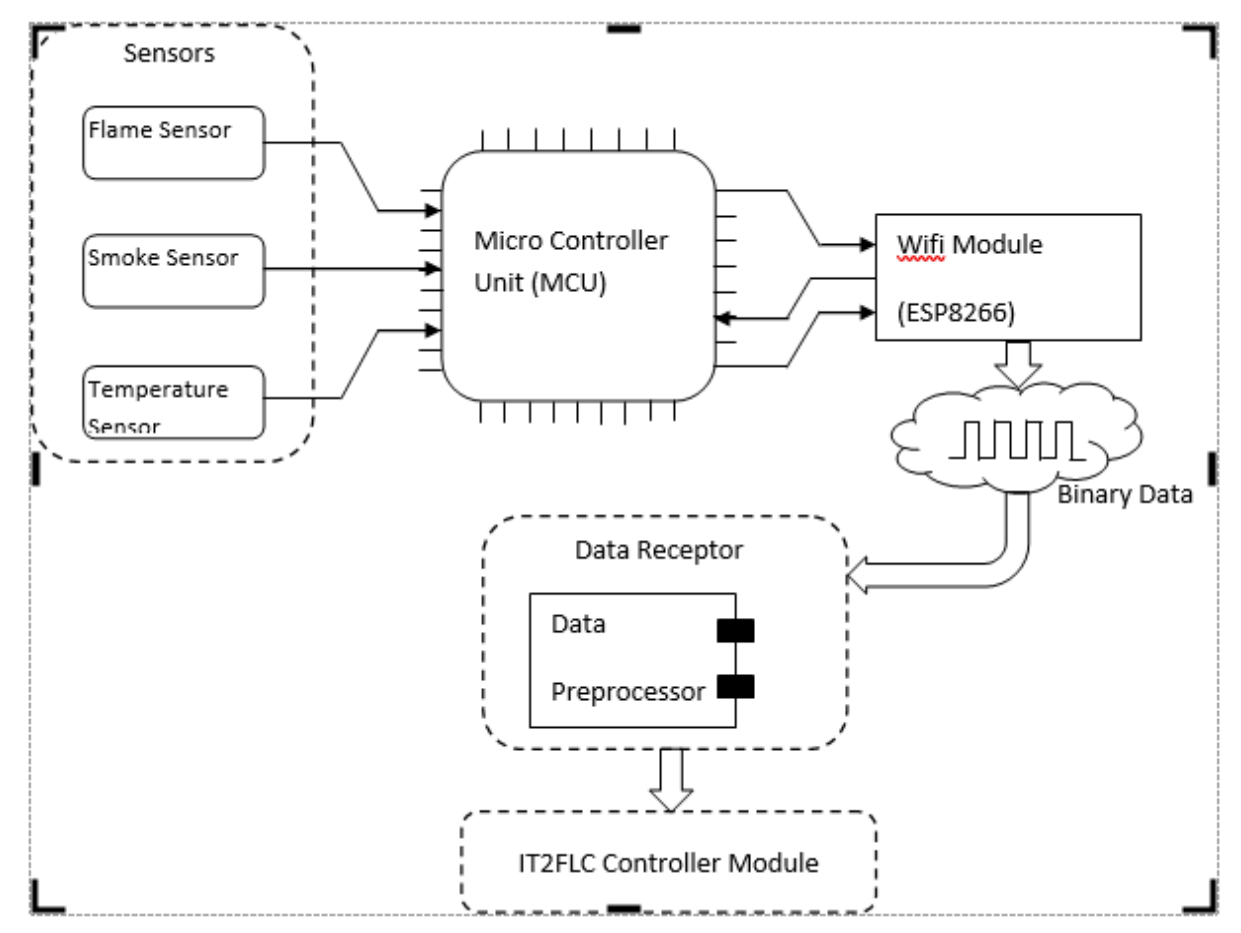

Figure 1: A Framework for Fire Outbreak Detection Based on Interval Type-2 Fuzzy Logic.

The components of fire outbreak detection system based on interval type-2 fuzzy logic algorithm are described below;

a. Sensors: In this paper, sensors are used to measure environmental parameters related to fire outbreak. The different types of sensors employed in this work are - DHT11 Temperature sensor is used to measure temperature, MQ-2 Smoke sensor is used to measure the amount of 
smoke in the air, and LM393 flame sensor is used to measure light intensity. These sensors provide a source of input values to the system. The sensors used in this work are depicted in figures 2,3 and 4 .

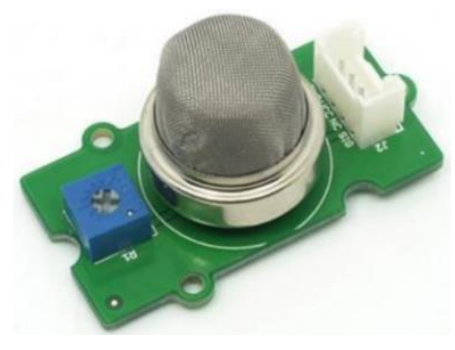

Figure 2: MQ-2 Smoke Sensor

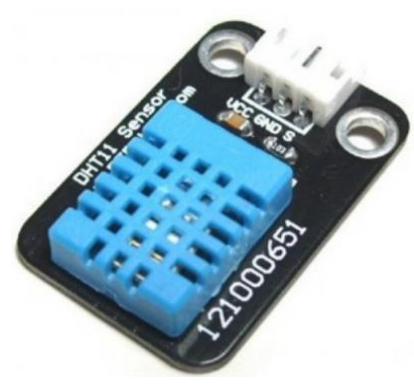

Figure 3: DHT11 Temperature Sensor

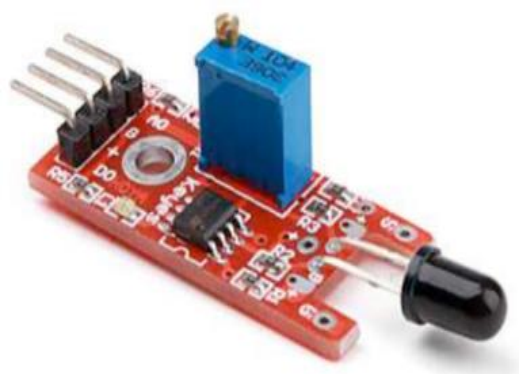

Figure 4: LM393 Flame Sensor

b. Micro CONTROLler Unit (MCU):

The Arduino Nano V3.0 micro chip is used to interface all the system peripherals (sensors, WIFI module). The MCU serves as the brain of the data capture device. Its main function is to read parameter values from the connected sensors and send same to the IT2-Fuzzy Logic module via an ESP8266 WIFI module. The Arduino board is presented in Figure 5;

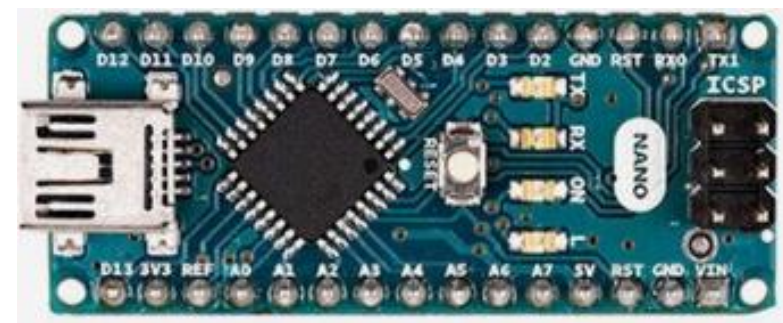

Figure 5: Arduino Nano V3.0 board

c. Data Receptor:

The Data receptor is a software module. It sends HTTP request for data to the ESP8266 WIFI module and accepts a response in the form of "HTTP Response Text". The format "HTTP $<\mathrm{html}>$ Response Text" is presented below;

\footnotetext{
$<$ head $><$ title $>$ FODCD Data $</$ title $></$ head $>$

$<$ body $>$ *temp,smoke,flame\#

The following heuristic algorithm is used by the Data Receptor to read and extract the environmental parameters (i.e temperature, smoke, and flame values) from the "HTTP Response Text"; 
algorithm Preprocess(msg):Arr[]

begin:

Define temp, smoke, flame;

Define dataSize N;

Define indexOfAsteric indx1, indexOfAsh indx2;

Define substringVariablesubStr;

Define array Variable Arr[];

//find the position of char *

Loop for $\mathrm{i}=0$ to $\mathrm{N}$

Let $\mathrm{x}=\operatorname{charAt}(\mathrm{i})$

If $\mathrm{x}==$ “*”

indx $1=\mathrm{i}$;

end if

break;

end for

//find the index of char \#

Loop for $\mathrm{j}=0$ to $\mathrm{N}$

$$
\begin{aligned}
& \text { Let } \mathrm{y}=\operatorname{charAt}(\mathrm{j}) \\
& \text { if } \mathrm{y}==\text { "\#” } \\
& \\
& \quad \text { Indx } 2=\mathrm{j} ; \\
& \text { break; } \\
& \text { end if }
\end{aligned}
$$

end for

//extract data substring and split to an array

if( indx $1>-1$ AND indx2 > -1)

subStr $=$ subString $($ ind $x 1$, ind $x 2)$;

Arr[] = split(subStr, “,”);

return Arr[];

end if

end

\section{d. Controller Module:}

The IT2FL Controller Module maps the crisp input gotten from the three sensors into a defuzzified value used in predicting the occurrence of fire outbreak. The controller module is implemented as an android application.

\section{Fire Outbreak Data Capture device}

The fire outbreak data capture (FODCD) is a device developed for the purpose of this work. This device is comprised of - Temperature Sensor, Smoke Sensor, Flame Sensor and an Arduino Microprocessor board. The Fire outbreak data capture device provides the input parameter values used in testing the performance of this system. The schematic diagram in Figure 6 shows the hardware components used in this work, their pin connection, and relationship between each of the components. 


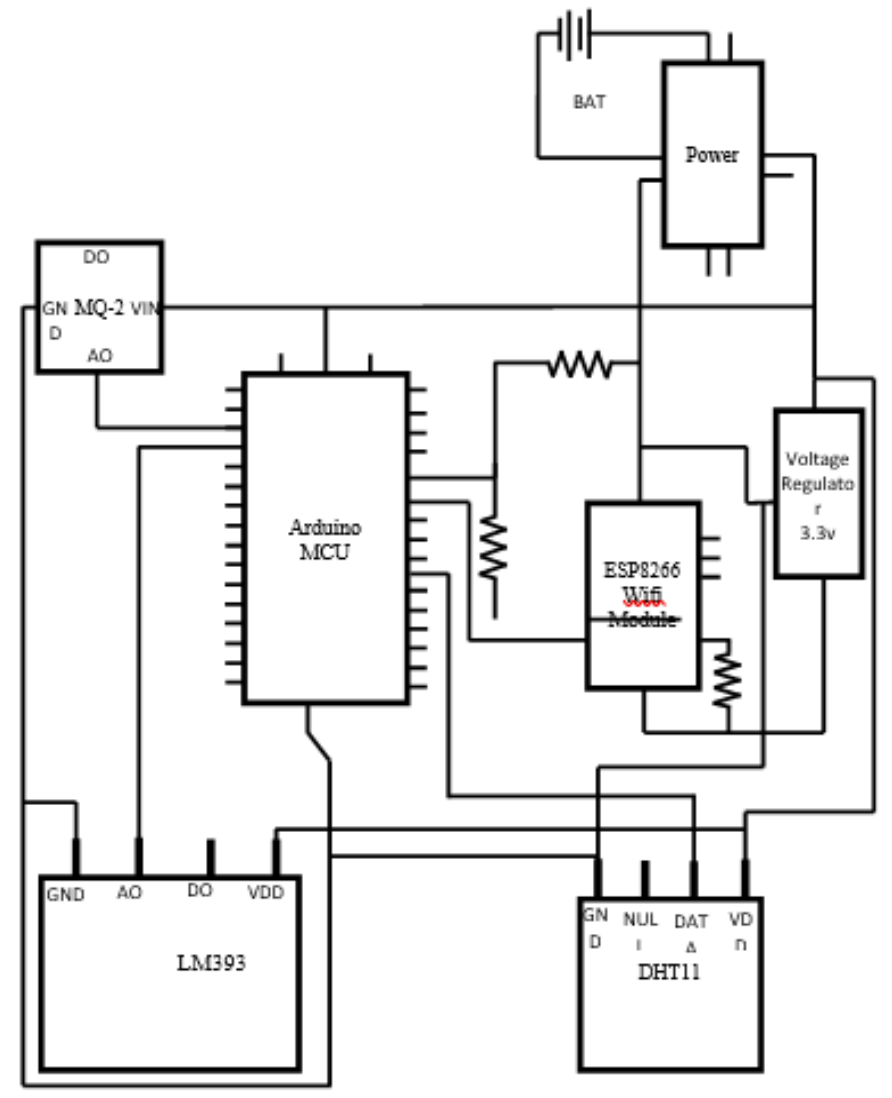

Figure 6: Schematic diagram of fire outbreak data capture device

The pins of the schematic diagram is described in Table I;

Table I: Schematic Symbol Description

\begin{tabular}{ll}
\hline PIN Name & Description \\
\hline GND & Ground PIN \\
AO & Analog Output PIN \\
DO & Digital Output PIN \\
VDD & Power Supply PIN \\
DATA & Data PIN \\
VIN & Voltage In PIN (Supply Voltage) \\
\hline
\end{tabular}

The device prototype is designed based on the schematic diagram above. Breadboard design is used to connect the necessary components of the device together. This helps in testing and debugging of the device before the final soldering is done. The prototype of the fire outbreak data capture device is presented in Figure 7; 


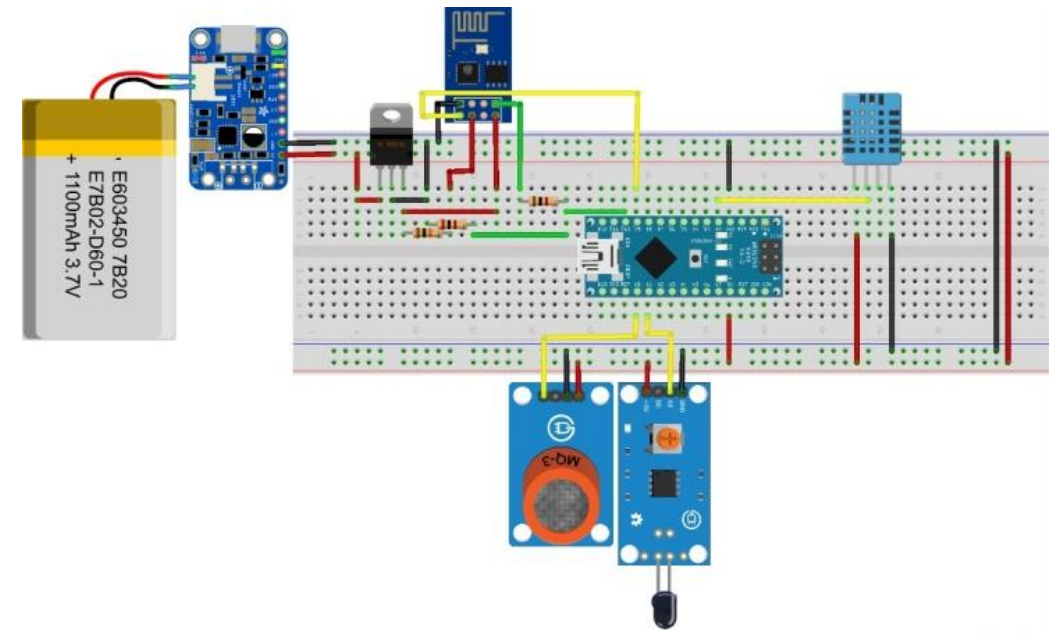

Figure 7: Prototype of the Fire Outbreak Data Capture Device

\section{Interval Type-2 Fuzzy Logic Algorithm for Fire Outbreak DETECTION}

This work uses the interval type-2 fuzzy logic (Liang and Mendel, 2010) controller adopted from Khule and Jangle (2017). The interval type-2 fuzzy logic model used in this work is presented in Figure 8;

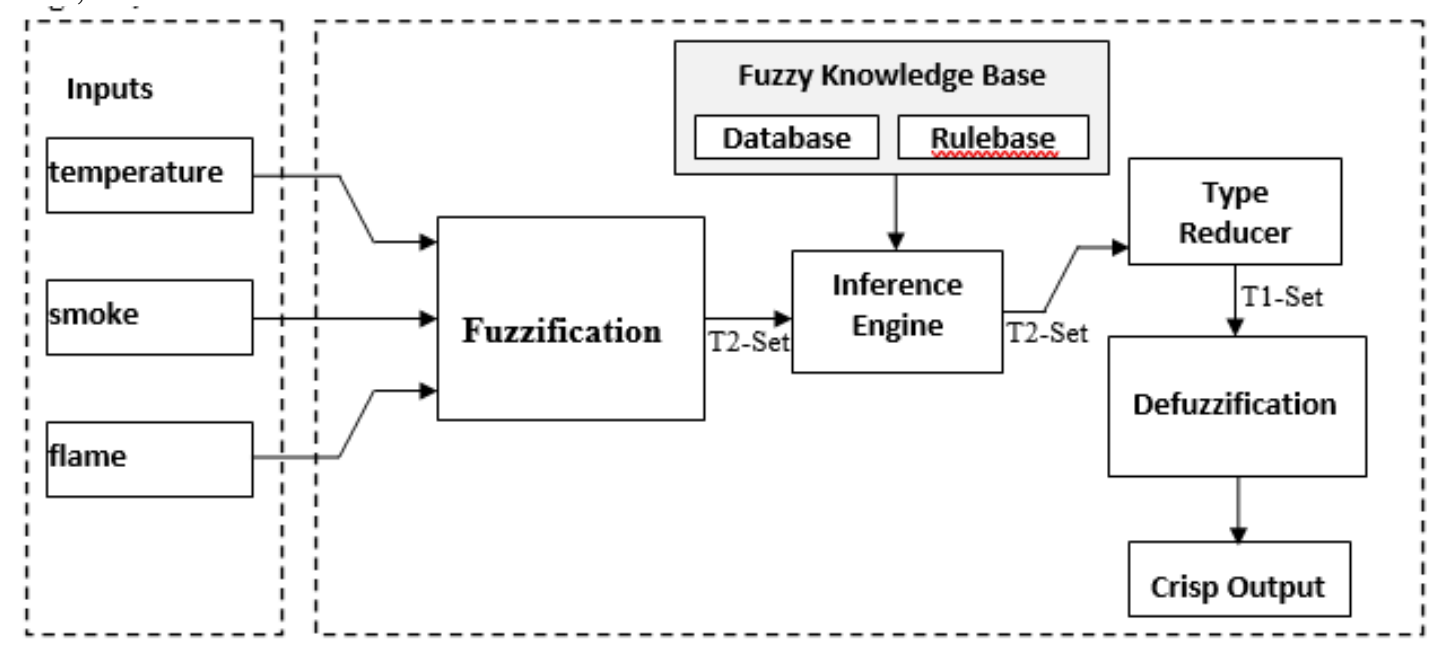

Figure 8: Interval Type-2 Fuzzy Logic Controller for Fire Outbreak Detection

The following components constitute the type-2 fuzzy logic model used in this work;

i. Fuzzification Module: this module maps the crisp input (fire outbreak parameter values) to a type-2 fuzzy set using gaussian membership function.

ii. Inference Engine: this module evaluates the rules in the knowledge base against type-2 fuzzy set gotten from Fuzzification module, to produce another type-2 fuzzy set.

iii. Type Reducer: Type reducer uses Karnik-Mendel algorithm to reduce an interval type-2 fuzzy set to type-1 fuzzy set. 
iv. Defuzzification Module: it maps the fuzzy set produced by type reducer to a crisp output using center of gravity Defuzzification method.

v. Fuzzy Knowledge Base: This is a database of rules (rules are generated from experts' knowledge) to be used by the inference engine.

vi. Membership Function: This is a mathematical equation that helps the fuzzification module convert the crisp input into a fuzzy set. This work uses the Gaussian membership functions.

The fire outbreak detection system is based on the three linguistic variables (parameters) Temperature, Smoke, and Flame. The linguistic variables and their universe of discourse are presented in Table II.

Table II: Universe of Discourse

\begin{tabular}{lll}
\hline Linguistic Variable & Universe of Discourse & Description \\
\hline Temp & {$[0,50]$} & Temperature(input) \\
Smoke & {$[0,100]$} & Smoke(input variable) \\
Flame & {$[0,1023]$} & Flame (input variable) \\
FireOutbreak & {$[0,1]$} & Output variable \\
\hline
\end{tabular}

This work is based on Gaussian membership function which is a mathematical expression used in the conversion of crisp input into fuzzy set. The Gaussian membership function is presented in Equations 1,2 and $3 ;$

$f(x)=e^{\frac{-1}{2}\left(\frac{x-c}{\sigma}\right)^{2}}, \sigma \in\left[\sigma_{1}, \sigma_{2}\right]$ and $c \in\left[c_{1}, c_{2}\right]$

$\underline{\mu}_{\bar{A}_{i m}}\left(x_{i}\right)=e^{\frac{-1}{2}\left(\frac{x_{i}-c_{i m}}{\sigma_{1, i m}}\right)^{2}}, \underline{\mu}_{\bar{A}}(x)=N\left(c, \sigma_{1} ; x\right)$

$\bar{\mu}_{\bar{A}_{i m}}\left(x_{i}\right)=e^{\frac{-1}{2}\left(\frac{x_{i}-c_{i m}}{\bar{\sigma}_{2, i m}}\right)^{2}}, \bar{\mu}_{\bar{A}}(x)=N\left(c, \sigma_{2} ; x\right)$

Where:

$c_{1}, c_{2}$ - are the centers of the lower and upper Gaussian membership functions respectively.

$\sigma_{1}, \sigma_{2}-$ are the variance (or width) of the lower and upper membership functions

$e$-is the exponential function

$x_{i}-$ is the input vector

$\bar{\mu}_{\bar{A}_{i m}}\left(x_{i}\right)$ - is the degree of membership of input $x_{i}$ in variable $i$ of linguistic term $m$.

The membership function partitioning that defines the linguistic terms for each linguistic variable as well as their ranges is presented in Table III, IV, V, and VI. The values $(\sigma, c)$ controls the shape of the membership function. Each membership function used in this work has two (2) control points $(\sigma, c)$, called the variance $(\sigma)$ and center $(c)$.

Table III: Membership function partitioning for temperature

\begin{tabular}{lll}
\hline TERMS & LOWER MF $(\boldsymbol{\sigma}, \boldsymbol{c})$ & UPPER MF $(\boldsymbol{\sigma}, \boldsymbol{c})$ \\
\hline Verylow & $3.834,0$ & $4.982,0$ \\
Low & $1.373,15.34$ & $2.37,15.34$ \\
Medium & $2.449,25.2$ & $3.81,25.2$ \\
High & $2.777,40$ & $4.173,40$ \\
Veryhigh & $3.45,50$ & $4.721,50$ \\
\hline
\end{tabular}


Table IV: Membership function partitioning for smoke

\begin{tabular}{lll}
\hline TERMS & LOWER MF $(\sigma, c)$ & UPPER MF $(\sigma, c)$ \\
\hline Verylow & $7.01,0$ & $9.22,0$ \\
Low & $5.53,27.76$ & $7.44,27.76$ \\
Medium & $5.532,50$ & $7.607,50$ \\
High & $4.908,67.95$ & $6.94,67.95$ \\
Veryhigh & $10.7,100$ & $13.54,100$ \\
\hline
\end{tabular}

Table V: Membership function partitioning for flame

\begin{tabular}{lll}
\hline TERMS & LOWER MF $(\sigma, c)$ & UPPER MF $(\sigma, c)$ \\
\hline Low & 239,0 & $287.3,0$ \\
Medium & $60.51,756$ & $87.17,756$ \\
High & $56.2,1023$ & $84.56,1023$ \\
\hline
\end{tabular}

Table VI: Membership function partitioning for "FireOutbreak"

\begin{tabular}{lll}
\hline TERMS & LOWER MF $(\sigma, c)$ & UPPER MF $(\sigma, c)$ \\
\hline Verylow & $0.0785,0$ & $0.1059,0$ \\
Low & $0.05459,0.3134$ & $0.08,0.3134$ \\
Medium & $0.05553,0.50$ & $0.08002,0.50$ \\
High & $0.05418,0.6887$ & $0.08,0.6887$ \\
Veryhigh & $0.1027,1$ & $0.1322,1$ \\
\hline
\end{tabular}

The interval type-2 fuzzy logic membership function plots for this system are presented in Figure (9) to Figure (12);

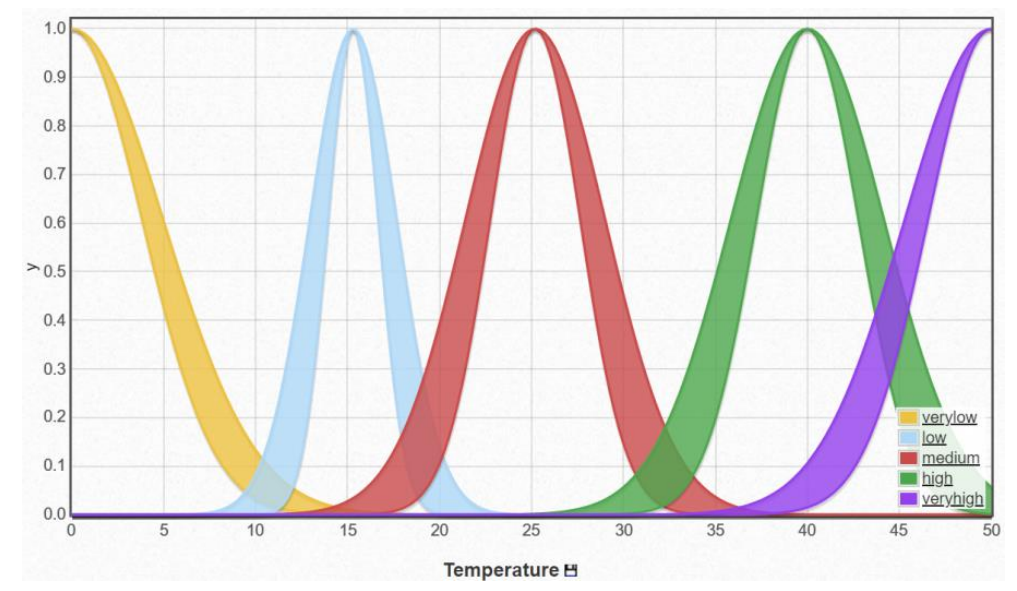

Figure 9: Membership function plot for temperature 
International Journal on Soft Computing, Artificial Intelligence and Applications (IJSCAI), Vol.8, No.3, August 2019

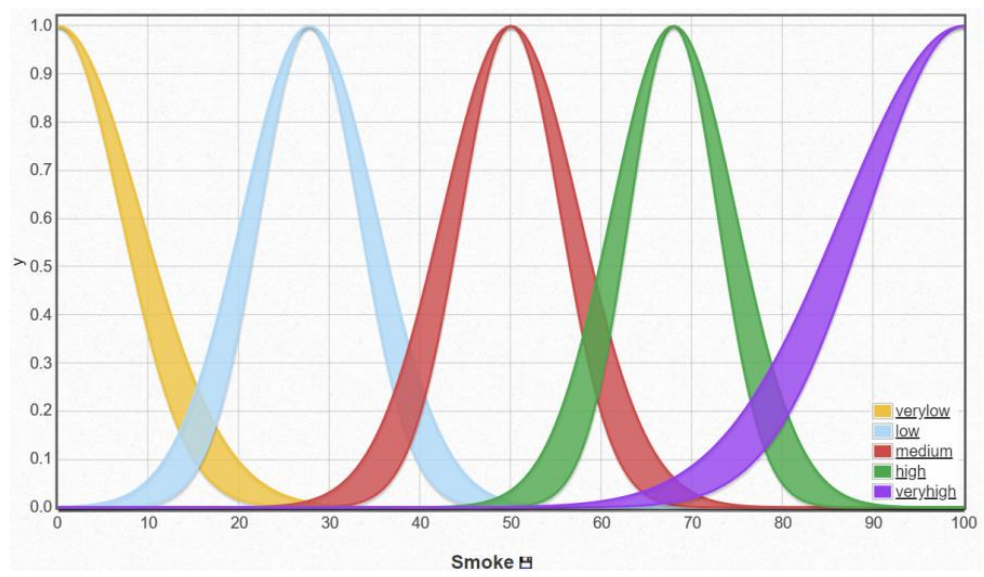

Figure 10: Membership function plot for smoke

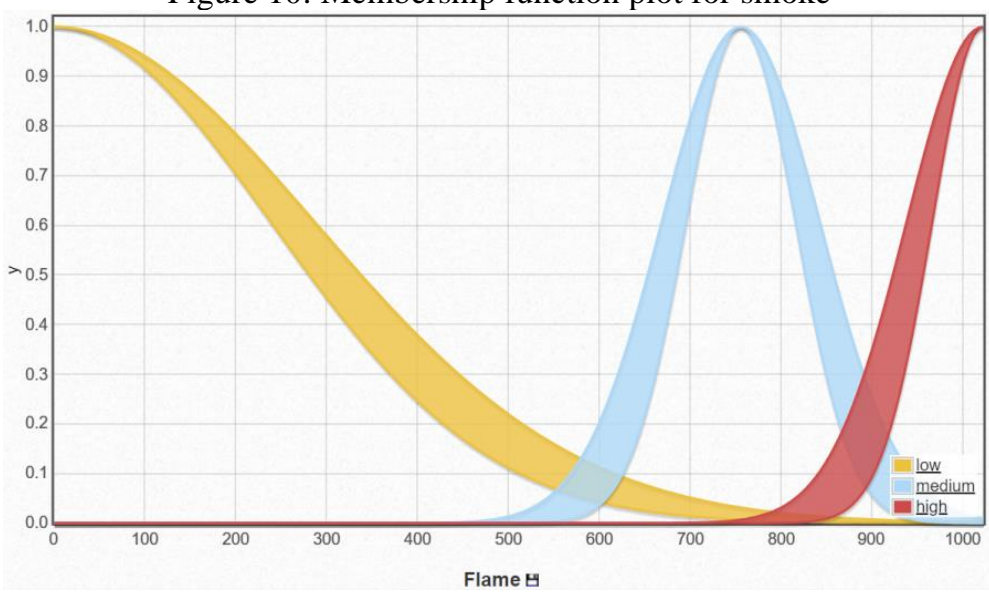

Figure 11: Membership function plot for Flame

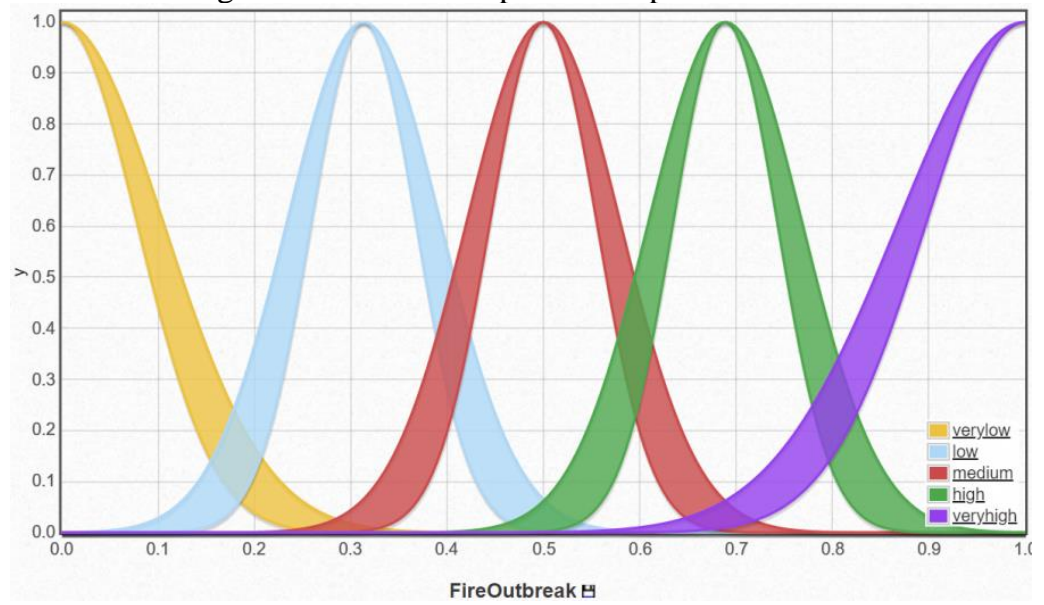

Figure 12: Membership function plot for fireOutbreak

The interval type-2 fuzzy rules defined for this system is a conditional statement in the form: $R^{l}: I F x_{1}$ is $\tilde{F}_{1}^{l}$ and ... $x_{p}$ is $\tilde{F}_{p}^{l}$ THEN $y$ is $\tilde{G}_{1}^{l}$ Where: 
$l:$ is the rule number $l=1, \ldots, M$

$x_{1}$ : is the linguistic variable

$\tilde{F}^{l}:$ is the linguistic term

In this system, fuzzy rules are defined using the standard form as;

IF temp IS Low AND smoke is Low AND flame is VeryLow THEN FireOutbreak is VeryLow.

Where temp, smoke and flame are the linguistic variables while Low and VeryLow are linguistic values (terms) determined by fuzzy sets on the universe of discourse temp, smoke, flame and fireOutbreak respectively. The number of rules used in this system is computed as the Number of Terms in temp * Number of Terms in smoke * Number of Terms in flame. The centroid of the consequent set $\left[\underline{y}^{1}, \bar{y}^{1}\right]$ (Karnik and Mendel, 2001), is calculated using the model;

$\underline{\mu}_{\bar{B}}(y)=\underline{\mu}_{F}(x) * \mu_{G}(y) \quad \forall y \in Y_{d}$

$\overline{\bar{\mu}}_{\bar{B}}(y)=\overline{\bar{\mu}}_{F}(x) * \overline{\bar{\mu}}_{G}(y) \quad \forall y \in Y_{d}$

The table below presents the subset of the fuzzy rules used in this system;

Table VII: Fuzzy Logic Rules

\begin{tabular}{lllll}
\hline S/N & Temperature & Smoke & Flame & FireOutbreak \\
\hline 1 & VL & VL & L & VL \\
2 & VL & VL & M & VL \\
3 & VL & VL & H & VL \\
4 & VL & L & L & VL \\
5 & VL & L & M & VL \\
6 & VL & L & H & L \\
7 & VL & M & L & VL \\
8 & VL & M & M & L \\
9 & VL & M & H & L \\
10 & VL & H & L & L \\
\hline
\end{tabular}

A Mamdani type inference mechanism is used to evaluate the fuzzy rules against the fuzzy set calculated from their respective membership functions. The Mamdani inference mechanism (Mamdanni and Assilian, 1975) is presented in Equations 7, 8 and 9.

$F^{i}\left(x^{\prime}\right)=\left[\underline{f}^{i}\left(x^{\prime}\right), \bar{f}^{i}\left(x^{\prime}\right)\right] \equiv\left[\underline{f}^{i}, \bar{f}^{i}\right]$

$\underline{f}^{i}\left(x^{\prime}\right)=\underline{\mu}_{\tilde{F}_{1}^{i}\left(x^{\prime}\right)} * \ldots * \underline{\mu}_{\widetilde{F}_{\mathrm{i}}^{i}}\left(x p^{\prime}\right)$

$\bar{f}^{i}\left(x^{\prime}\right)=\bar{\mu}_{\tilde{F}_{1}^{i}\left(x^{\prime}\right)} * \ldots * \bar{\mu}_{\tilde{F}_{\mathrm{i}}^{i}\left(x p^{\prime}\right)}$

Where;

$\left[\underline{f}^{i}, \bar{f}^{i}\right]$ - is the firing antecedent set.

$\underline{\mu}_{\tilde{F}_{\mathrm{i}}^{i}\left(x^{\prime}\right)}$ - is the fuzzy set of the lower membership function

$\bar{\mu}_{\tilde{F}_{\mathrm{i}}^{i}\left(x^{\prime}\right)}$ - is the fuzzy set of the upper membership function

\subsection{TYPE REDUCTION}

This work employs Karnik Mendel type reduction algorithm (Mendel, 2001) to reduce the interval 
type-2 fuzzy set to a type-1 fuzzy set for use by the Defuzzification module. This algorithm seeks to find switch points $(\mathrm{L}, \mathrm{R})$ and then leftmost point $\left(y_{l}\right)$ and the rightmost point $\left(y_{r}\right)$ determined by;

$$
\begin{aligned}
& y^{L} \leq y_{l} \leq y^{L+1} \\
& \bar{y}^{R} \leq y_{r} \leq \bar{y}^{R+1}
\end{aligned}
$$

Where $\underline{y}^{n}$ and $\bar{y}^{n}$ have been sorted in ascending order, respectively.

The Karnik Mendel algorithm to find the switch points are as follows;

KM Algorithm for Computing $\boldsymbol{y}_{\boldsymbol{l}}$

Step 1: sort $\underline{y}^{n}(\mathrm{n}=1,2, \ldots, \mathrm{N})$ in increasing order and call the sorted $\underline{y}^{n}$ by the same, but now $\underline{y}^{1} \leq$ $\underline{y}^{2} \leq \cdots \leq \underline{y}^{N}$. Match the weights $F^{n}\left(X^{\prime}\right)$ with their respective $\underline{y}^{n}$ and renumber them so that their index corresponds to the renumbered $\underline{y}^{n}$.

Step 2:Initialize $f^{n}$ by setting

$f^{n}=\frac{f^{n}+\bar{f}^{n}}{2} \quad n=1,2, \ldots, N$

And then compute

$y=\frac{\sum_{n=1}^{R} \underline{y}^{n} f^{n}}{\sum_{n=1}^{R} f^{n}}$

Step 3: Find the switch point $k(1 \leq k \leq N-1)$ such that $\underline{y}^{k} \leq y \leq \underline{y}^{k+1}$

Step 4: Set $f^{n}=\left\{\begin{array}{l}\bar{f}^{n}, n \leq k \\ \underline{f}^{n}, n>k\end{array}\right.$

And compute

$y^{\prime}=\frac{\sum_{n=1}^{N} \underline{y}^{n} f^{n}}{\sum_{n=1}^{N} f^{n}}$

Step 5: Check if $y^{\prime}=y$. If yes, stop and set $y_{l}=y$ and $\mathrm{L}=\mathrm{k}$. if no, go to Step 6 .

Step 6: Set $y=y^{\prime}$ and go to Step 3

KM Algorithm for Computing $\boldsymbol{y}_{\boldsymbol{r}}$

Step 1: sort $\bar{y}^{n}(\mathrm{n}=1,2, \ldots, \mathrm{N})$ in increasing order and call the sorted $\bar{y}^{n}$ by the same, but now $\bar{y}^{1} \leq$ $\bar{y}^{2} \leq \cdots \leq \bar{y}^{N}$. Match the weights $F^{n}\left(X^{\prime}\right)$ with their respective $\bar{y}^{n}$ and renumber them so that their index corresponds to the renumbered $\bar{y}^{n}$.

Step 2:Initialize $f^{n}$ by setting

$f^{n}=\frac{f^{n}+\bar{f}^{n}}{2} \quad n=1,2, \ldots, N$

And then compute

$y=\frac{\sum_{n=1}^{N} \bar{y}^{n} f^{n}}{\sum_{n=1}^{N} f^{n}}$

Step 3: Find the switch point $k(1 \leq k \leq N-1)$ such that $\bar{y}^{k} \leq y \leq \bar{y}^{k+1}$

Step 4: Set $f^{n}=\left\{\begin{array}{l}f^{n}, n \leq k \\ \bar{f}^{n}, n>k\end{array}\right.$

And compute

$y^{\prime}=\frac{\sum_{n=1}^{N} \bar{y}^{n} f^{n}}{\sum_{n=1}^{N} f^{n}}$

Step 5: Check if $y^{\prime}=y$. If yes, stop and set $y_{r}=y$ and $\mathrm{R}=\mathrm{k}$. if no, go to Step 6 . 
Step 6: Set $y=y^{\prime}$ and go to Step 3

The algorithm to find the leftmost and the rightmost points are given in Equation (12) and Equation (13) (Mendel, 2001), (Mendel and Wu, 2010), (Wu and Mendel, 2009);

$y_{l}=\underset{L \in[1, N-1]}{\min } \frac{\sum_{n=1}^{L} \bar{f}^{n} \underline{y}^{n}+\sum_{n=L+1}^{N} \underline{f}^{n} \underline{y}^{n}}{\sum_{n=1}^{L} \bar{f}^{n}+\sum_{n=L+1}^{N} \underline{f}^{n}}$

$y_{r}=\underset{R \in[1, N-1]}{\max } \frac{\sum_{n=1}^{R} \underline{f}^{n} \bar{y}^{n}+\sum_{n=R+1}^{N} \bar{f}^{n} \bar{y}^{n}}{\sum_{n=1}^{R} \underline{f}^{n}+\sum_{n=R+1}^{N} \bar{f}^{n}}$

Defuzzification of the fuzzy set produced by Karnik Mendel's algorithm is done using the center of gravity defuzzification algorithm presented in Equation (14);

$y_{k}(x)=\frac{y_{l}+y_{r}}{2}$

\section{RESUltS AND DiscuSSION}

The Fire Outbreak Data Capture Device (FODCD) used in capturing the temperature, smoke and flame parameter values for this work is presented in Figure 13 and Figure 14. The FODCD was used in this work to capture environmental parameters such as temperature, smoke and flame.

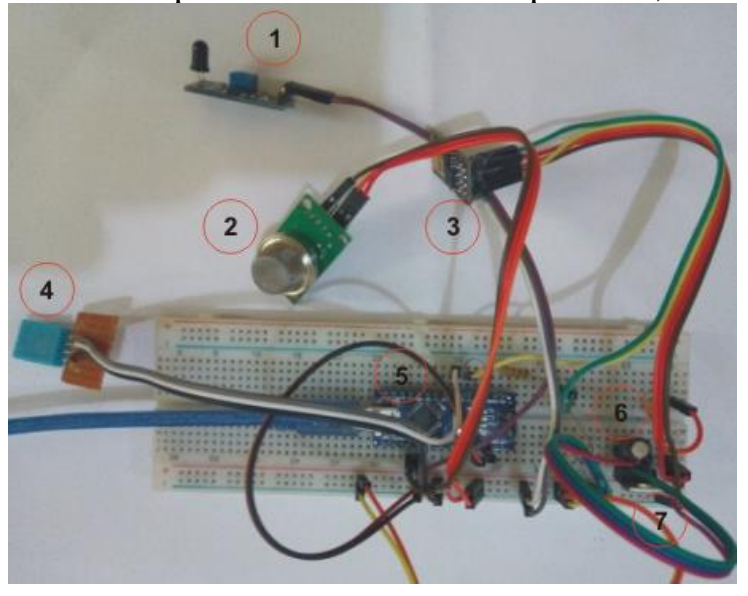

Figure 13: Breadboard of FODCD

Figure 13 shows the components of the FODCD connected together using a Breadboard. Component labeled (1) is the Flame sensor, (2) is the Smoke sensor, (3) is the WIFI Module, (4) is the Temperature sensor, (5) is the Arduino Board, (6) is the Capacitor, (7) is the Voltage regulator to put the voltage in the acceptable range. 


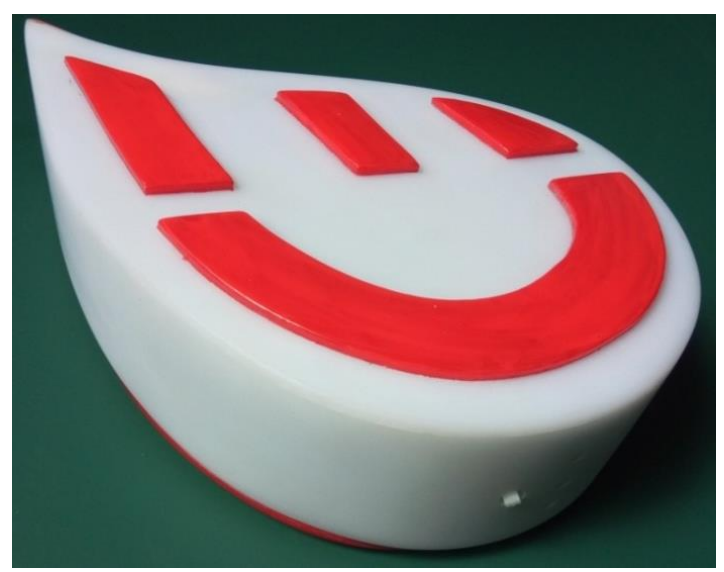

Figure 14: Fire Outbreak Data Capture Device

Figure 14 shows the acrylic package of the FODCD. This package is used to house all the sensors, arduino board, and the WIFI module. The FODCD is powered by pressing the switch (white colored) located at the bottom of the device. On power-on, the FODCD broadcast a WIFI signal named "FODCD Device" to the controller device which is an Android device running the "Interval Type-2 Controller for Fire Outbreak Detection" application. The user (Android Device) is required to connect to the broadcasted network in other to start receiving environmental parameters (i.e. temperature, smoke and flame values) that is used for fire outbreak detection.

Using the Fire Outbreak Data Capture Device (FODCD), 30 data points were obtained. This data is presented in Table VIII;

Table VIII: Obtained Parameter Values

\begin{tabular}{|c|c|c|c|}
\hline $\mathrm{S} / \mathrm{N}$ & TEMP & SMOKE & FLAME \\
\hline 1 & 27.263 & 52.441 & 546.63 \\
\hline 2 & 49.873 & 82.65 & 900.66 \\
\hline 3 & 40.657 & 77.86 & 762.95 \\
\hline 4 & 36.076 & 68.233 & 450.37 \\
\hline 5 & 19.826 & 37.872 & 389.94 \\
\hline 6 & 37.674 & 71.794 & 565.7 \\
\hline 7 & 21.81 & 36.77 & 397.36 \\
\hline 8 & 32.906 & 60.597 & 414.79 \\
\hline 9 & 17.881 & 28.9 & 269.92 \\
\hline 10 & 29.021 & 53.883 & 507.25 \\
\hline 11 & 18.115 & 32.369 & 287.57 \\
\hline 12 & 17.591 & 29.446 & 228.11 \\
\hline 13 & 39.609 & 64.403 & 706.88 \\
\hline 14 & 35.586 & 64.151 & 498.78 \\
\hline 15 & 35.558 & 58.21 & 567.57 \\
\hline 16 & 44.852 & 78.745 & 916.79 \\
\hline 17 & 37.807 & 65.478 & 731.85 \\
\hline 18 & 25.894 & 46.496 & 399.69 \\
\hline 19 & 21.793 & 41.868 & 334.87 \\
\hline 20 & 17.358 & 28.658 & 281.47 \\
\hline 21 & 29.892 & 58.262 & 564.8 \\
\hline 22 & 23.802 & 42.706 & 434.35 \\
\hline 23 & 16.939 & 32.034 & 247.68 \\
\hline 24 & 18.532 & 29.671 & 352.26 \\
\hline 25 & 20.981 & 36.558 & 388.42 \\
\hline 26 & 19.764 & 34.761 & 287.94 \\
\hline 27 & 30.29 & 54.489 & 452.74 \\
\hline 28 & 26.908 & 51.535 & 523.7 \\
\hline 29 & 21.221 & 39.101 & 312.77 \\
\hline 30 & 35.374 & 67.543 & 484.69 \\
\hline
\end{tabular}


International Journal on Soft Computing, Artificial Intelligence and Applications (IJSCAI), Vol.8, No.3, August 2019

These input parameters can be visualized using the graphs in Figures 15,16,17 and 18;

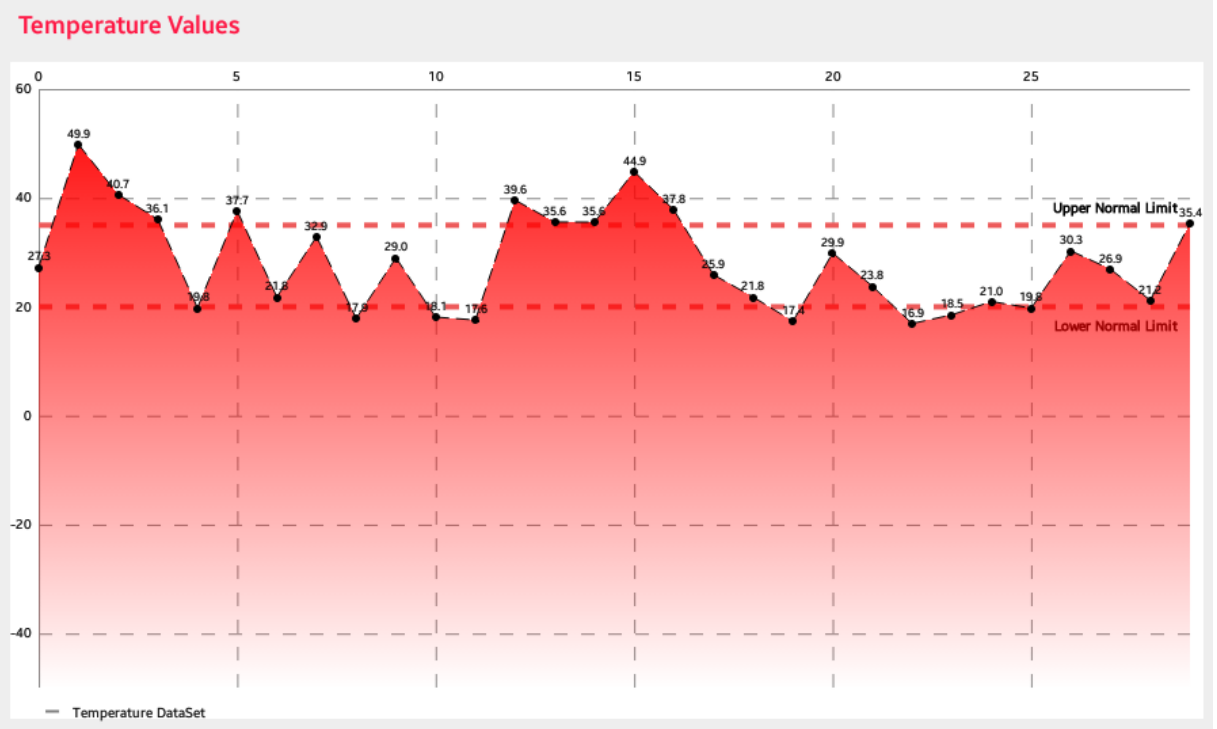

Figure 15: Input Values for Temperature

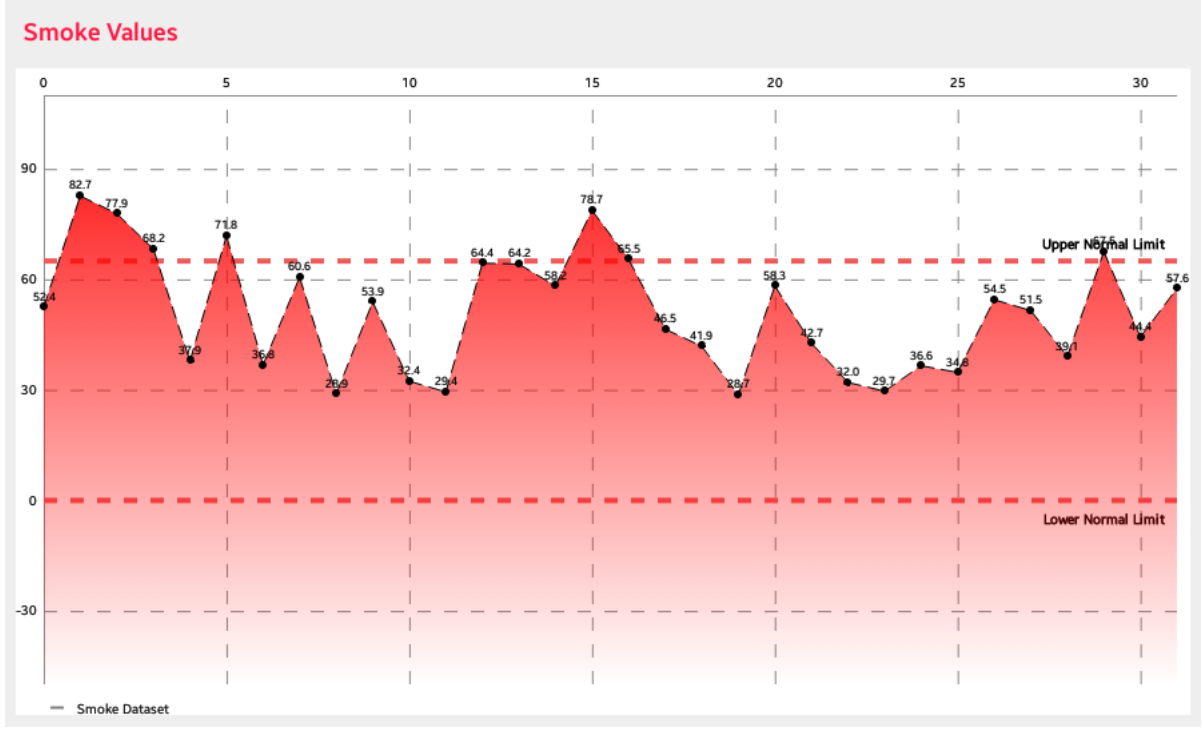

Figure 16: Input Values for Smoke 
International Journal on Soft Computing, Artificial Intelligence and Applications (IJSCAI), Vol.8, No.3, August 2019

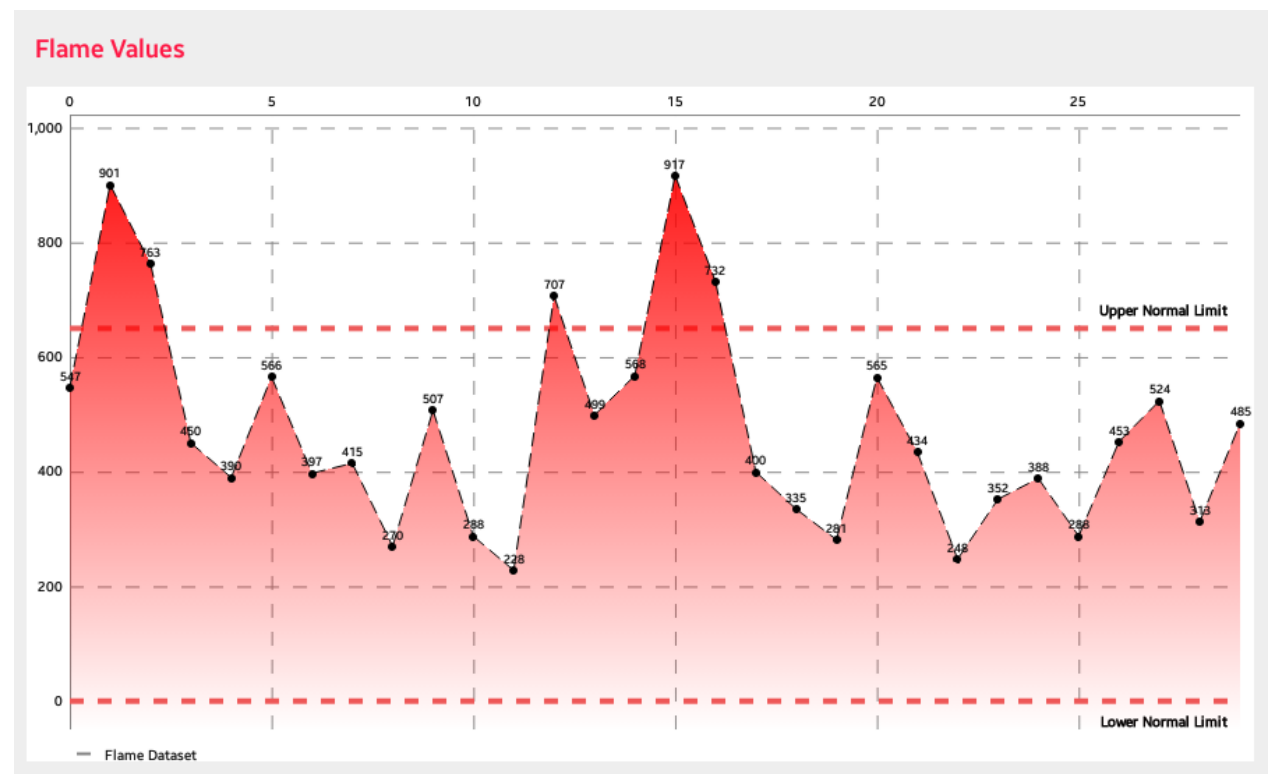

Figure 17: Input Values for Flame

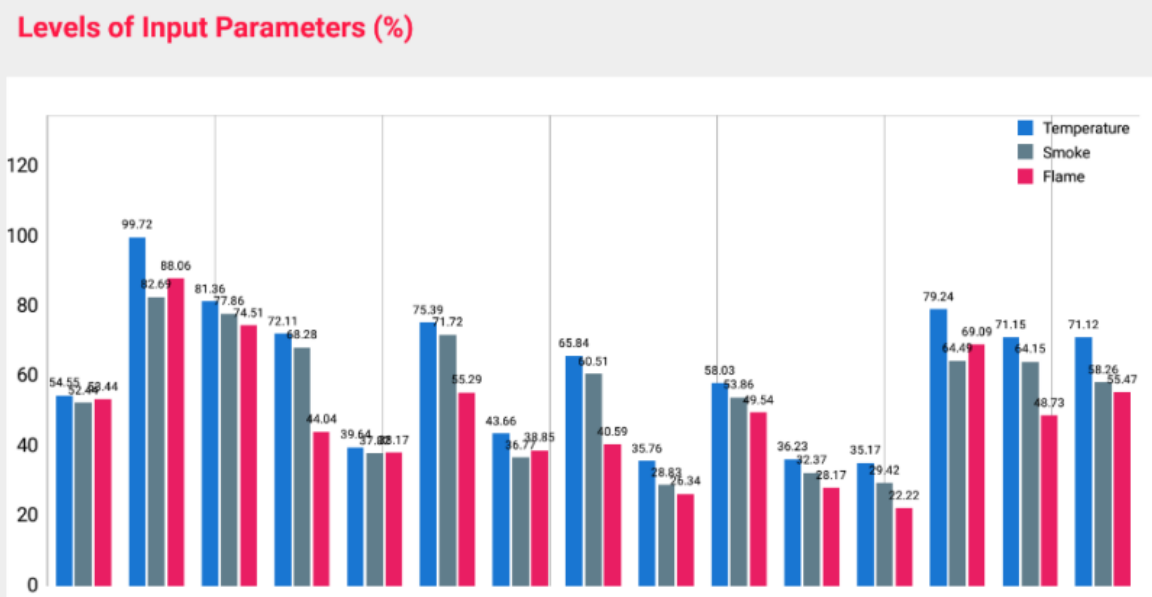

Figure 18: Levels of Input Parameter Values

The effect of the input parameters (temperature, smoke and flame) on the fire outbreak is presented in Figure 19; 


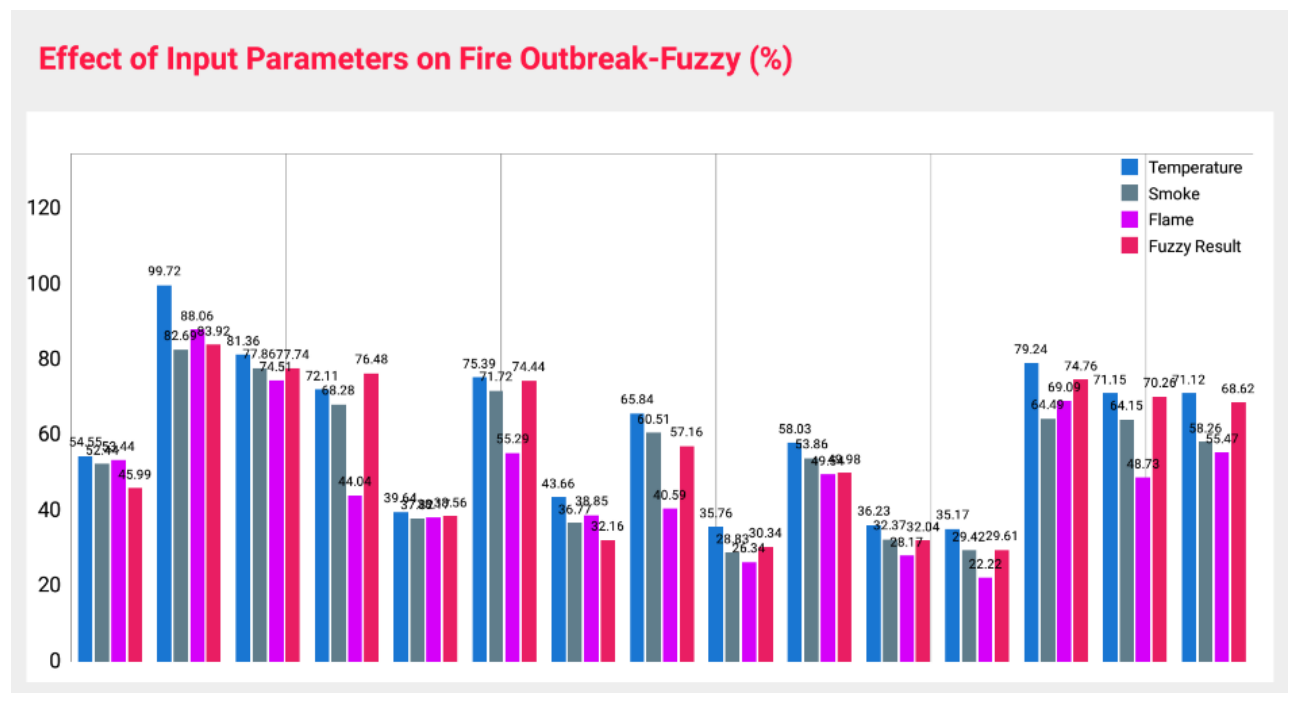

Figure 19: Effect of the input parameters on fire outbreak

The result of fuzzifying the above input parameters using the interval type-2 fuzzy logic algorithm is presented in Table IX.

Table IX: Fuzzy Fire Outbreak Prediction Result

\begin{tabular}{|c|c|c|c|c|c|}
\hline $\mathrm{S} / \mathrm{N}$ & TEMP & SMOKE & FLAME & IT2FL Result & IT2FL Decision \\
\hline 1 & 27.263 & 52.441 & 546.63 & 0.4598612 & No Fire Outbreak \\
\hline 2 & 49.873 & 82.65 & 900.66 & 0.8398821 & Fire Outbreak \\
\hline 3 & 40.657 & 77.86 & 762.95 & 0.777972 & No Fire Outbreak \\
\hline 4 & 36.076 & 68.233 & 450.37 & 0.7641621 & No Fire Outbreak \\
\hline 5 & 19.826 & 37.872 & 389.94 & 0.3857935 & No Fire Outbreak \\
\hline 6 & 37.674 & 71.794 & 565.7 & 0.7445189 & No Fire Outbreak \\
\hline 7 & 21.81 & 36.77 & 397.36 & 0.3217344 & No Fire Outbreak \\
\hline 8 & 32.906 & 60.597 & 414.79 & 0.5715717 & No Fire Outbreak \\
\hline 9 & 17.881 & 28.9 & 269.92 & 0.3037507 & No Fire Outbreak \\
\hline 10 & 29.021 & 53.883 & 507.25 & 0.4995942 & No Fire Outbreak \\
\hline 11 & 18.115 & 32.369 & 287.57 & 0.3203094 & No Fire Outbreak \\
\hline 12 & 17.591 & 29.446 & 228.11 & 0.2961141 & No Fire Outbreak \\
\hline 13 & 39.609 & 64.403 & 706.88 & 0.7473185 & No Fire Outbreak \\
\hline 14 & 35.586 & 64.151 & 498.78 & 0.702256 & No Fire Outbreak \\
\hline 15 & 35.558 & 58.21 & 567.57 & 0.6866619 & No Fire Outbreak \\
\hline 16 & 44.852 & 78.745 & 916.79 & 0.8396478 & Fire Outbreak \\
\hline 17 & 37.807 & 65.478 & 731.85 & 0.7414769 & No Fire Outbreak \\
\hline 18 & 25.894 & 46.496 & 399.69 & 0.4509105 & No Fire Outbreak \\
\hline 19 & 21.793 & 41.868 & 334.87 & 0.4180752 & No Fire Outbreak \\
\hline 20 & 17.358 & 28.658 & 281.47 & 0.287153 & No Fire Outbreak \\
\hline 21 & 29.892 & 58.262 & 564.8 & 0.5608679 & No Fire Outbreak \\
\hline 22 & 23.802 & 42.706 & 434.35 & 0.4323183 & No Fire Outbreak \\
\hline 23 & 16.939 & 32.034 & 247.68 & 0.2746664 & No Fire Outbreak \\
\hline 24 & 18.532 & 29.671 & 352.26 & 0.3233087 & No Fire Outbreak \\
\hline 25 & 20.981 & 36.558 & 388.42 & 0.3546594 & No Fire Outbreak \\
\hline 26 & 19.764 & 34.761 & 287.94 & 0.3863372 & No Fire Outbreak \\
\hline 27 & 30.29 & 54.489 & 452.74 & 0.588482 & No Fire Outbreak \\
\hline 28 & 26.908 & 51.535 & 523.7 & 0.457647 & No Fire Outbreak \\
\hline 29 & 21.221 & 39.101 & 312.77 & 0.3711399 & No Fire Outbreak \\
\hline 30 & 35.374 & 67.543 & 484.69 & 0.7374535 & No Fire Outbreak \\
\hline
\end{tabular}


International Journal on Soft Computing, Artificial Intelligence and Applications (IJSCAI), Vol.8, No.3, August 2019

The following metrices are used to evaluate the performance of this system - True Positive (TP), False Positive (FP), True Negative (TN), False Negative (FN), True Positive Rate (TPR), False Positive Rate (FPR), Precision, Accuracy, and Error rate. The performance analysis of fire outbreak detection system is presented in Table $\mathrm{X}$

Table X: Performance Evaluation of the System

\begin{tabular}{|c|c|c|c|c|c|c|c|c|c|c|}
\hline $\mathbf{S} / \mathbf{N}$ & Threshold & $\mathbf{T P}$ & $\mathbf{F P}$ & $\mathbf{F N}$ & TN & TPR (Recall) & FPR & $\begin{array}{l}\text { PRECESI } \\
\text { ON }\end{array}$ & $\begin{array}{l}\text { ACCURA } \\
\text { CY }\end{array}$ & $\begin{array}{l}\text { ERROR } \\
\text { RATE }\end{array}$ \\
\hline 1 & 0.6 & 3 & 7 & 0 & 20 & 1 & 0.2593 & 0.3 & $76.67 \%$ & 0.23 \\
\hline 2 & 0.7 & 3 & 6 & 0 & 21 & 1 & 0.2222 & $\begin{array}{r}0.33333333 \\
3\end{array}$ & $80 \%$ & 0.2 \\
\hline 3 & 0.8 & 2 & 0 & 1 & 27 & 0.667 & 0 & 1 & $96.67 \%$ & 0.03 \\
\hline 4 & 0.85 & 0 & 0 & 3 & 27 & 0 & 0 & 0 & $90 \%$ & 0.1 \\
\hline 5 & 0.9 & 0 & 0 & 3 & 27 & 0 & 0 & 0 & $90 \%$ & 0.1 \\
\hline
\end{tabular}

Table $\mathrm{X}$ shows the effect of threshold on the system performance. This effect can be visualized in Figure 20;

Accuracy - Fuzzy Result

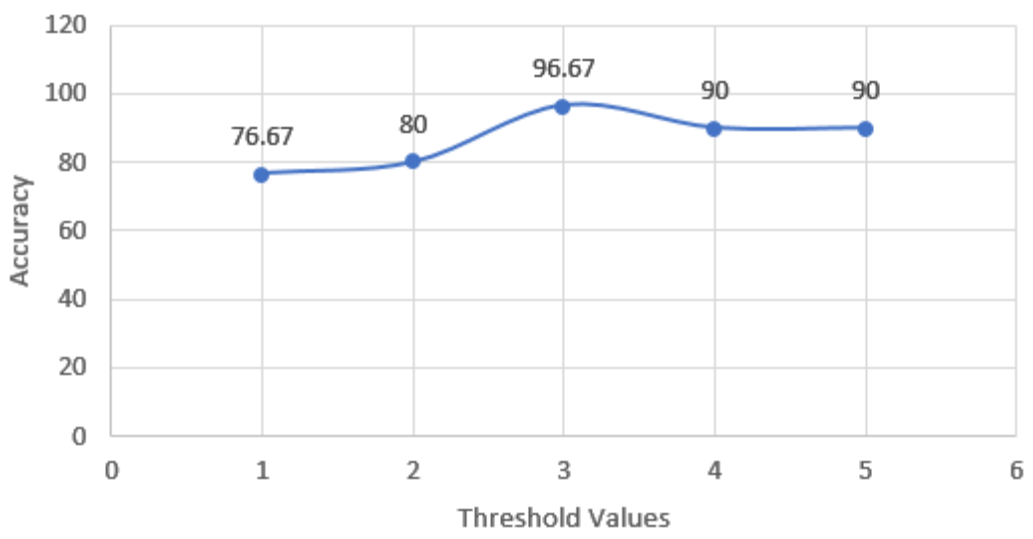

Figure 20: Effect of threshold values on system accuracy

Error Rate - Fuzzy Result

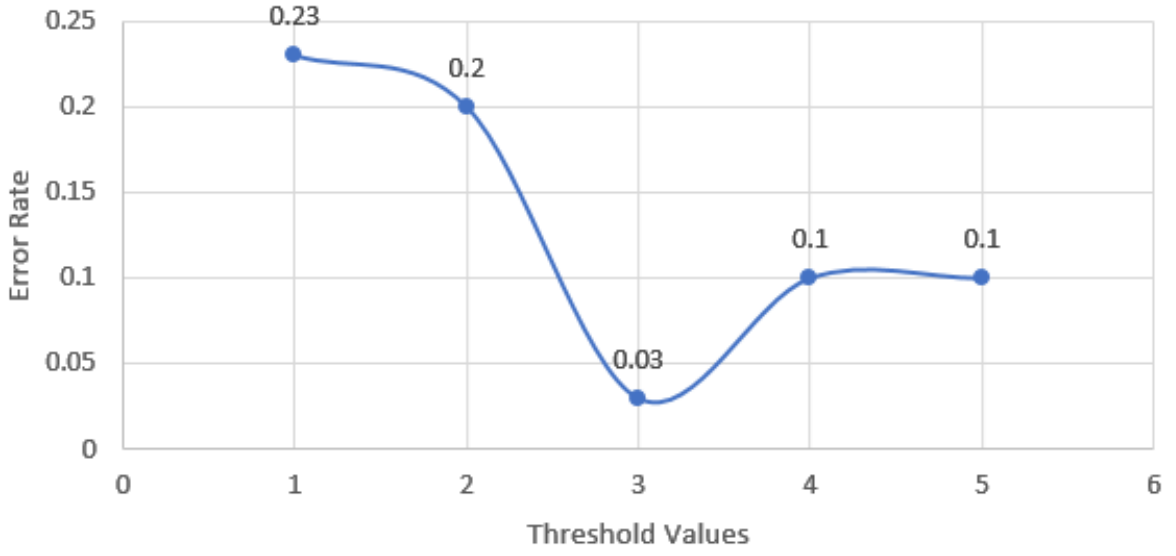

Figure 21: The System Error Rate

Figure 20 and Figure 21, show that any threshold value below 0.8 or above 0.85 negatively affects the performance of the system. Hence the best threshold for this system is between 0.8 and 0.85 with an 
accuracy value of $90 \%$ and $96.67 \%$ respectively.

The result of this system shows that the choice of the threshold value can affect the system's performance; hence care must be taken when choosing the threshold value. The best threshold value is the one with zero percent $(0 \%)$ error rate and highest possible accuracy and a reduced false positive rate. This system has attained a better performance by using 0.825 as the threshold value. This threshold value can then be generalized to any data point with a varying accuracy between $90 \%$ and $96.67 \%$. The result of this system show that a framework for fire outbreak detection based on interval type-2 fuzzy logic is able to detect cases of fire outbreak with minimal error rate between $0.03 \%$ and $0.1 \%$.

\section{Conclusion}

A Framework for Fire Outbreak Detection Based on Interval Type-2 Fuzzy Logic has been able to proffer solution to the problems associated with fire outbreak by providing continuous monitoring of environmental changes that are responsible for fire outbreak such as Temperature, Smoke and Flame. The system is also able to detect cases of fire outbreak and take remedial actions such as notifying the house owner and then sending a distress text to the fire service with location information. The system can predict a case of fire outbreak with accuracy between $90 \%$ and $96.67 \%$ when the threshold value is set between an optimal range of 0.8 and 0.85 with a minimal error rate between $0.03 \%$ and $0.1 \%$. It is possible to detect and take remedial action during fire outbreak. These actions include the use of a Framework for Fire Outbreak Detection Based on Interval Type-2 Fuzzy Logic to promptly predict cases of fire outbreak.

When this is done, we can protect lives and properties as well as reduce the rate of depression resulting from fire disaster. The combination of sensors, fuzzy controller, and android system gives the best result to the problem of fire management.

\section{REFERENCES}

[1] Bezdek, J. (1993). Fuzzy models-what are they, and why? IEEE Trans. on Fuzzy Systems, volume 1, 1-5.

[2] Chu, X. J. (2010). Design of Fire Detection Algorithm Based on Fuzzy Logic and using Wireless Sensors. Canada: Ottawa-Carleton Institute of Electrical and Computer Engineering (OCIECE).

[3] Hagras, H. (2007). Type-2 FLCs: A new generation of fuzzy controllers, IEEE Computational Intelligence Magazine, volume 2, 30-43.

[4] Jantzen, J. (1999). Design Of Fuzzy Controllers. Denmark: Technical University of Denmark, Department of Automation.

[5] Karnik, N. N., Mendel, J. M. (2001). Centroid of a type-2 fuzzy set. Information Sciences, volume 132, 195-220.

[6] Khule, V., Jangle, N. N. (2017). Design and implementation of a fire and obstacle detection and control system using fuzzy logic with notification system to avoid automobile accidents. 10th International Conference on Recent Trends in Engineering Science and Management. India: Newton's School of Science and Technology, 307-313.

[7] Liang, Q., Mendel, J. M. (2000). Interval Type-2 Fuzzy Logic Systems: Theory and Design. IEEE Transaction on Fuzzy Systems, volume 8, 535-550.

[8] Mamdani, E. H., Assilian, S. (1975). An experiment in linguistic synthesis with a fuzzy logic controller. International Journal of Man-Machine Studies , 1-13. 
International Journal on Soft Computing, Artificial Intelligence and Applications (IJSCAI), Vol.8, No.3, August 2019

[9] Mendel, J. M. (1995). Introduction to Ruled-Based Fuzzy Logic Systems. California: University of South California.

[10] Mendel, J. M. (2001) Uncertain Rule-Based Fuzzy Logic Systems: Introduction and New Directions. Upper Saddle River, NJ: Prentice-Hall.

[11] Mendel, J. M., Wu, D. (2010). Perceptual Computing: Aiding People in Making Subjective Judgments. Hoboken, NJ: Wiley-IEEE Press.

[12] Mobin, M. A., Rafi, M., Islam, M. N. (2016). An Intelligent Fire Detection and Mitigation System Safe from Fire. International Journal of Computer Applications, Volume 133, 1-6.

[13] Ross, T. J. (2004). Fuzzy Logic with Engineering Applications, Second Edition. John Wiley \& Sons.

[14] Saeed, F.; Paul, A.; Rehman, A.; Hong, W.H.; Seo, H. IoT-Based Intelligent Modeling of Smart Home Environment for Fire prevention and Safety. J. Sens. Actuator Netw. 2018, 7, 11.

[15] Sarwar, B., Bajwa, I. S., Shabana, R., Ramzan, B. and Kausar, M. (2018). Design and Application of Fuzzy Logic Based Fire Monitoring and Warning Systems for Smart Buildings. Symmetry, Volume 10, Issue 615, 1-24, doi:10.3390/sym10110615.

[16] Sharma, D. (2016). Implementation of Artificial Neural Fuzzy Inference System in a Real Time Fire Detection Mechanism. International Journal of Computer Applications, Volume 146, 31-36.

[17] Singh, D., Sharma, N., Gupta, M. and Sharma, S. (2017). Development of System for Early Fire Detection using Arduino UNO. Int. J. Eng. Sci. Comput., 7, 10857-10860.

[18] Sowah, R. (2014). A Review on Forest Fire Detection Techniques. International Journal of Distributed Sensor Networks, 2-8.

[19] Sowah, R.; Ampadu, K.O.; Ofoli, A.; Koumadi, K.; Mills, G.A.; Nortey, J. (2016). Design and Implementation of a Fire Detection and Control System for Automobiles using Fuzzy Logic. In Proceedings of the IEEE Industry Applications Society Annual Meeting, Portland, OR, USA, 2-6 October 2016.

[20] Wu, D., Mendel, J. M. (2009). Enhanced Karnik-Mendel Algorithms. IEEE Trans. on Fuzzy Systems, volume. 17, 923-934.

[21] Wu, D., Tan, W. W. (2006). Genetic learning and performance evaluation of type-2 fuzzy logic controllers. Engineering Applications of Artificial Intelligence, volume 19, 829-841.

[22] Xin, S. Y. (2012). Multi-objective Flower Algorithm for Optimization. Procedia Computer Science , volume. 18, 155-173.

[23] Zadeh, L. A. (1965). Fuzzy sets and systems. Journal of Information Control., 338-353. 